\title{
Principais características da raiz de cenoura na perspectiva de agentes da cadeia produtiva
}

\author{
Silvia S Onoyama ${ }^{1}$; Giovani Olegario da Silva²; Antônio W Moita ${ }^{\text {; }}$ Jairo V Vieira $^{1}$; Carlos Alberto Lopes ${ }^{1}$; \\ Geraldo da S e Souza ${ }^{3}$ \\ 1Embrapa Hortaliças, C. Postal 218, 70351-970 Brasília-DF; silvia@cnph.embrapa.br; moita@cnph.embrapa.br; jairo@cnph.embrapa. \\ br; clopes@cnph.embrapa.br. ㄹEmbrapa Hortaliças-SNT, C. Postal 317, 89460-000 Canoinhas-SC; olegario@cnph.embrapa.br (autor cor- \\ respondente); ${ }^{3}$ Embrapa Sede, SGE, 70770-901 Brasília-DF; geraldo.souza@embrapa.br
}

\section{RESUMO}

Procurou-se verificar quais são as características mais importantes da raiz de cenoura sob a ótica dos agentes da cadeia produtiva de cenoura. Para tanto, foram realizadas visitas e entrevistas com produtores, empresas de semente, vendedores de insumos, lavadores, processadores, atacadistas e gerentes de supermercados, comparando-se esses resultados com os da pesquisa de percepção de consumidores de supermercados do Distrito Federal. Em ambos os casos, utilizou-se questionário estruturado com perguntas fechadas nas quais os entrevistados classificaram os atributos segundo notas que variaram de 1 (nenhuma importância) a 5 (muito importante). Para verificar a ordenação de importância dos atributos e os contrastes das médias, utilizou-se o método estatístico de análise de dados categóricos. $\mathrm{O}$ resultado da pesquisa indicou que os consumidores estão preocupados com valores nutricionais, textura, cor externa e sabor da cenoura. Já os demais agentes da cadeia atentaram mais para as características relacionadas com a padronização, com a coloração externa e com o ombro verde. Foram detectadas diferenças de percepção entre os agentes da cadeia, em especial dos consumidores, para os quais foram observadas diferenças nas respostas em função do gênero, idade, grau de escolaridade, estado civil e localização do supermercado. Estes resultados são muito importantes para o direcionamento de projetos de pesquisa com cenoura, que precisa atender as exigências tanto do setor produtivo quanto dos consumidores.

\begin{abstract}
Main carrot root attributes based on stake holders perceptions

The present investigation aimed at elucidating the carrot attributes which stake holders view as the most important. A research was carried out with carrot growers, seed companies, supplier salespersons, washers, food processors, wholesalers and supermarket managers and was compared to the perception of consumers from supermarkets located in the Federal District of Brazil. Data sets were collected through structured interviews with closed questions about the importance of a range of attributes on a 1 (not important) to 5 (very important) scale. Statistic method based on categorical analysis was used to rank the attributes and calculate the average contrasts. The survey indicated that, according to consumers, nutritional value, texture, external color and taste are the most important attributes. In contrast, the other stake holders pay more attention to characteristics related to shape uniformity, external color and green shoulder. In addition, differences of perception by specific chain agents were detected, in special to the consumers group, affected by gender, age, educational level, marital status and supermarket location. These results are very important for directing carrot research projects, which must meet the productive sector and consumers requirements.
\end{abstract}

Keywords: Daucus carota L., plant breeding, market research.

Palavras-chave: Daucus carota L., melhoramento, pesquisa de mercado.

(Recebido para publicação em 26 de novembro de 2010; aceito em 6 de julho de 2011)

(Received on November, 26 2010; accepted on July 6, 2011)

\begin{abstract}
A cenoura (Daucus carota L.) é umas das hortaliças com maior destaque no cenário nacional, correspondendo a $6 \%$ da produção total de hortaliças (Vilela \& Henz, 2000). Segundo Vilela \& Borges (2008), a cenoura é posta em evidência principalmente por apresentar elevada capacidade de geração de emprego e renda em todos os segmentos de sua cadeia produtiva durante o ano inteiro. Em face disso, instituições de pesquisa como a Embrapa e empresas produtoras de semente dão ênfase a programas de melhoramento de cenoura em seu portfólio de pesquisa. No Brasil, antes de 1981, as cultivares de cenoura
\end{abstract}

disponíveis no mercado eram estrangeiras, principalmente originárias da França, dos Estados Unidos e do Japão e voltadas para o cultivo no inverno. A partir daí, em programas de melhoramento nacionais, foram desenvolvidas novas cultivares adaptadas às condições edafoclimáticas brasileiras, que possibilitaram significativo aumento de produtividade e elevada redução nos custos finais da produção e produtividade elevada, resultando em alta rentabilidade e retornos econômicos compensadores (Vilela \& Borges, 2008).

Por outro lado, observa-se que tais cultivares atendem preferencialmente às necessidades dos produtores e atacadistas, já que os atributos aos quais tradicionalmente é dada maior importância são resistência a doenças, alto vigor, produtividade, redução do custo de produção e uniformidade de raiz. Mesmo em outras culturas, os projetos de pesquisa atentam principalmente para os problemas de cultivo da planta e os atributos-chave para os clientes finais têm sido pouco considerados como alvos das pesquisas de melhoramento genético (Matsuura et al., 2004).

Entretanto, Blackwell et al. (2005); Ciccantelli \& Magidson (1993), Clark \& Wheelwright (1993) e Cooper (1999) 
ressaltam que o sucesso no desenvolvimento de novos produtos depende do entendimento das necessidades do mercado. Na perspectiva de Blackwell et al. (2005), um produto novo deve satisfazer as necessidades, desejos e expectativas de seus principais consumidores, sejam intermediários ou finais.

No intuito de dar continuidade ao sucesso dos programas de melhoramento da Embrapa, percebeu-se a necessidade de integrar as demandas dos diversos atores da cadeia produtiva no desenvolvimento de novas cultivares. Segundo Brennan \& Byerlee (1991), o tempo necessário para o desenvolvimento e lançamento de uma cultivar é de aproximadamente uma década; por isso, no olhar de Kosina et al. (2007), deve-se assegurar o alinhamento dos objetivos da pesquisa de longo prazo com as necessidades dos atores da cadeia que envolve produtores, processadores, atacadistas, consumidores, dentre outros para que a nova cultivar seja inserida com sucesso no mercado.

Seguindo essa perspectiva, pesquisadores do programa de melhoramento de cenoura da Embrapa Hortaliças prospectaram as necessidades do mercado para auxiliar na programação da sua pesquisa da Embrapa Hortaliças. Neste sentido, o presente trabalho tem por objetivo mapear os principais atributos da raiz de cenoura junto aos principais atores da cadeia produtiva dessa hortaliça no Brasil por meio de entrevistas estruturadas, seguindo os preceitos de Malhotra (2001). De acordo com o autor, o método baseia-se num interrogatório ao participante, aos quais fazem perguntas sobre seu comportamento, intenções, atitudes, percepções, motivações, características demográficas e estilos de vida.

\section{MATERIAL E MÉTODOS}

A pesquisa adotou o método de entrevista com os agentes da cadeia para obter as principais características da raiz de cenoura. O questionário da coleta de dados foi elaborado por meio de mesas redondas com especialistas da Embrapa. Realizaram-se cinco reuniões com pesquisadores das áreas de melhoramento, pós-colheita, fitopatologia, sócio-economia e transferência de tecnologia, vol- tados à cultura da cenoura, para discutir quais atributos do produto comercial que podem ser percebidos pelos agentes da cadeia. Ao final, chegou-se aos seguintes atributos: a) atributos relacionados à padronização: peso, diâmetro, formato e comprimento; b) atributos relacionados à aparência: intensidade da cor externa, intensidade da cor interna, ombro verde e lisura de raiz; c) atributos relacionados à parte sensorial: doçura, amargor e crocância; d) vitamina (valor nutricional); e f) preço.

Os agentes da cadeia foram divididos em dois grupos. O primeiro grupo totalizou 66 entrevistados, que envolveu produtores das principais regiões produtivas, lavadores, comerciantes, empresas de sementes, atacadistas, processadoras, representantes de insumos e de supermercados, pertencentes às seguintes localidades: Marilândia do Sul-PR, Londrina-PR, Caxias do Sul-RS, São José do Rio Pardo-SP, Mogi das Cruzes-SP, Piedade-SP, São Paulo-SP, São Gotardo-MG e Irecê-BA. Os supermercados estão localizados no Distrito Federal, sendo dois com abrangência nacional, três com destaque na região do Centro-Oeste e três com venda somente no Distrito Federal.

A formação do grupo 1 foi considerada não probabilística intencional, sendo que os casos escolhidos pelo pesquisador são julgados típicos da população na qual ele está interessado, supondo assim que os erros na seleção tenderão a contrabalançar-se (Mattar, 1997).

O grupo 2 foi constituído por 500 potenciais consumidores de cenoura de cinco supermercados do Distrito Federal, localizados nas regiões administrativas de Ceilândia, Taguatinga, Asa Norte, Riacho Fundo e Recanto das Emas. A opção por esses locais foi baseada nos dados sobre estratificação das regiões administrativas em cinco grupos de acordo com a classe de renda: a população da Asa Norte está inserida no primeiro grupo de renda, seguida por Taguatinga, Riacho Fundo, Ceilândia e Recanto das Emas (Codeplan, 1997). A escolha dos supermercados foi aleatória e realizaram-se 100 entrevistas em cada um deles.

Para o cálculo do tamanho do grupo, o intervalo de confiança foi de 95\%, e o tamanho dos grupos foi calculado a partir de uma amostra piloto de 100 consumidores do primeiro supermercado entrevistado, com a utilização dos preceitos de Mattar (1997).

As entrevistas estruturadas ocorreram na seção de hortifrutigranjeiros abordando, ao acaso, os consumidores de diferentes faixas etárias, grau de escolaridade, sexo e estado civil.

O período do trabalho abrangeu os meses de novembro de 2008 a fevereiro de 2009. Os dias das entrevistas ocorreram às terças, quartas, quintas e sábados, dias nos quais ocorria maior demanda por produtos hortifrutigranjeiros.

Os itens do questionário foram mensurados na escala Likert de cinco pontos, variando de 1 (nenhuma importância) a 5 (muito importante). Primeiramente, realizou-se tratamento descritivo dos dados que envolveu cálculo das médias e dos desvios-padrões e distribuição do grau de importância dos atributos. Para verificar a ordenação de importância dos atributos, os contrastes das médias e a influência do perfil do agente da cadeia na avaliação dos atributos utilizou-se do procedimento CATMOD do SAS (Stokes et al., 2000), que analisou as características categóricas por meio de regressão múltipla e distribuição não normal.

\section{RESULTADOS E DISCUSSÃO}

Os consumidores foram predominantemente do público feminino, com $65,3 \%$. A faixa etária, dividida em cinco grupos, apresentou a seguinte distribuição: $17,4 \%$ com até 30 anos, $18,2 \%$ entre 31 a 40 anos, $24,8 \%$ entre 41 a 50 anos, $25,9 \%$ entre 51 a 60 anos e $13,7 \%$ com mais de 60 anos. A maioria dos consumidores deste grupo foi casada com $50,3 \%$, os solteiros corresponderam a $30,6 \%$ e viúvos, divorciados e outros abrangeram 19,1\%. O grau de escolaridade foi distribuído em quatro segmentos, dando destaque a $37,8 \%$ que possuem segundo grau completo (foi somado aos que estão cursando o ensino superior), seguido por $21,3 \%$ com primeiro grau incompleto, $20,7 \%$ com ensino superior completo (foi somado aos que já têm curso de pós-graduação) 
Tabela 1. Ranqueamento dos atributos de cenoura pelos agentes da cadeia dos grupos $1 \mathrm{e}$ 2 utilizando a técnica do CATMOD (ranking of the carrot attributes by the chain agents of the groups 1 and 2 using the technique of CATMOD). Brasília, Embrapa Hortaliças, 2009.

\begin{tabular}{lcclllc}
\hline \multicolumn{3}{c}{ Grupo 1 } & & & \multicolumn{3}{c}{ Grupo 2 } \\
\cline { 1 - 4 } Atributos & Média & $\begin{array}{c}\text { Desvio } \\
\text { padrão }\end{array}$ & & Atributos & Média & $\begin{array}{c}\text { Desvio } \\
\text { padrão }\end{array}$ \\
\hline Ombro verde & $4,81^{*}$ & 0,63 & & Vitamina & $4,50 \mathrm{a}^{* *}$ & 1,05 \\
Uniformidade & 4,73 & 0,85 & & Crocância & $4,32 \mathrm{~b}$ & 1,25 \\
Preço/custo & 4,58 & 0,68 & & Cor externa & $4,20 \mathrm{~b}$ & 1,19 \\
Cor externa & 4,51 & 0,99 & & Doçura & $3,98 \mathrm{c}$ & 1,34 \\
Lisura na raiz & 4,51 & 0,94 & & Preço & $3,92 \mathrm{~cd}$ & 2,76 \\
Diâmetro & 4,49 & 0,82 & & Cor interna & $3,89 \mathrm{~cd}$ & 1,38 \\
Comprimento & 4,42 & 0,94 & & Lisura na raiz & $3,77 \mathrm{~d}$ & 1,54 \\
Formato & 4,23 & 1,21 & & Diâmetro & $3,19 \mathrm{e}$ & 1,41 \\
Cor interna & 3,27 & 1,52 & & Comprimento & $3,16 \mathrm{e}$ & 1,45 \\
Peso & 3,06 & 1,50 & & Uniformidade & $3,13 \mathrm{e}$ & 1,55 \\
Crocância & 2,99 & 1,73 & & Formato & $2,86 \mathrm{f}$ & 1,49 \\
Amargor & 2,43 & 1,55 & & Amargor & $2,58 \mathrm{fg}$ & 1,71 \\
Doçura & 2,27 & 1,52 & & Peso & $2,51 \mathrm{gh}$ & 1,41 \\
Vitamina & 1,41 & 1,00 & & Ombro verde & $2,32 \mathrm{~h}$ & 1,62 \\
\hline
\end{tabular}

* Os contrastes não foram calculados porque a amostragem não é probabilística, portanto não se pode realizar inferência estatística (the contrasts were not calculated because the sample is not probabilistic, so cannot make statistical inference).**Médias seguidas de letras diferentes na coluna diferiram significativamente a $5 \%$ de probabilidade de erro (means followed by different letters in the column differ significantly at 5\% level of probability). Grupo 1: 66 agentes da cadeia de cenoura. Grupo 2: 500 consumidores do Distrito Federal (Group 1: 66 agents of the carrot chain. Group 2: 500 consumers of the Federal District).

e $20,1 \%$ com primeiro grau completo. Os respondentes que possuem filhos foram $82,5 \%$. A pesquisa foi realizada em supermercados, pois é crescente o comércio de hortaliças neste canal de comercialização (Vilela \& Henz, 2000).

Com a identificação das principais características da cenoura realizada através da análise de dados categóricos (Tabela 1), a ordenação dos atributos obedeceu à seguinte ordem: a) grupo 1: ombro verde, uniformidade, preço/ custo, cor externa, lisura da raiz, diâmetro, comprimento, formato, cor interna, peso, crocância, amargor, doçura e vitamina (valor nutricional); b) grupo 2: vitamina, crocância, intensidade da cor externa, doçura, preço, intensidade cor interna, lisura da raiz, diâmetro, comprimento, uniformidade da raiz, formato, amargor, peso e ombro verde.

A utilização deste tipo de classificação dos dados se deve ao fato de estes resultarem de observações de variáveis aleatórias (atributos) cujos valores possíveis são classificados em um conjunto
Ressalta-se que esses atributos são destacados pelos atacadistas, por serem valorados na tabela de classificação da cenoura da Ceagesp.

Por outro lado, os consumidores entendem como característica mais importante da cenoura o seu valor nutricional. De acordo com a percepção dos entrevistados, a cenoura é uma hortaliça de elevado valor nutritivo, fonte de vitamina A; confirmando com a literatura, que menciona valor nutricional como um dos atributos mais importantes na decisão de compra (Souza, 2005). Nas entrevistas, observou-se que o consumidor relacionava a cenoura a altos índices de beta-caroteno, independente do tipo de cultivar, haja vista que não há informações sobre tipos de cultivares nos supermercados.

Outras características priorizadas pelos consumidores foram crocância, intensidade da cor externa, doçura, preço e intensidade da cor interna e lisura da raiz. Essas características remetem ao aspecto e à questão sensorial do produto. Vale observar a importância dada pelo consumidor ao sabor da cenoura, mesmo sem experimentá-la durante a compra, bem como a relação entre crocância e firmeza do produto. Kader (2002) pondera que os atributos que mais influenciam o consumidor são sabor ou aroma, textura e aparência, uma vez que os consumidores se baseiam no visual e no que aparenta ter gosto bom.

Num segundo momento, através da ferramenta CATMOD, calcularam-se as diferenças de percepções dos consumidores. Vale ressaltar que não foi possível fazer inferência estatística entre as percepções dos agentes da cadeia porque a amostragem não é probabilística. Dentre os consumidores, detectaram-se diferenças entre as percepções de homens e mulheres para as características peso, formato, coloração interna e crocância (Tabelas 2a e 2b). Homens enfatizaram a padronização do produto, já as mulheres valoraram mais as características sensoriais e a textura. Na esfera do estado civil, os solteiros pontuaram vitamina como mais importante do que os casados. Para as demais características, a percepção foi a mesma.

Quanto ao grau de escolaridade, as pessoas com maior instrução formal 
Tabela 2a. Importância dos atributos da raiz de cenoura segundo o perfil dos agentes de cadeia do grupo 2 (importance of the carrot root attributes according to the profile of the chain agents of group 2). Brasília, Embrapa Hortaliças, 2009.

\begin{tabular}{lccccccc}
\hline Perfil & $\mathbf{U N}$ & $\mathbf{P E}$ & $\mathbf{D I}$ & $\mathbf{F O}$ & $\mathbf{C O}$ & $\mathbf{C E}$ & $\mathbf{C I}$ \\
\hline Sexo & $\mathrm{p}<0,05$ & $\mathrm{p}<0,05$ & $\mathrm{p}=0,51$ & $\mathrm{p}<0,05$ & $\mathrm{p}<0,05$ & $\mathrm{p}=0,91$ & $\mathrm{p}<0,05$ \\
Masculino & $3,39 \mathrm{a}$ & $2,69 \mathrm{a}$ & $3,24 \mathrm{a}$ & $3,12 \mathrm{a}$ & $3,37 \mathrm{a}$ & $4,22 \mathrm{a}$ & $3,69 \mathrm{a}$ \\
Feminino & $2,99 \mathrm{~b}$ & $2,40 \mathrm{~b}$ & $3,16 \mathrm{a}$ & $2,71 \mathrm{~b}$ & $3,02 \mathrm{~b}$ & $4,20 \mathrm{a}$ & $4,00 \mathrm{~b}$ \\
Escolaridade & $\mathrm{p}<0,05$ & $\mathrm{p}=0,35$ & $\mathrm{p}=0,11$ & $\mathrm{p}=0,58$ & $\mathrm{p}=0,52$ & $\mathrm{p}=0,30$ & $\mathrm{p}=0,26$ \\
$1^{\circ}$ grau incompleto & $3,58 \mathrm{a}$ & $2,72 \mathrm{a}$ & $3,38 \mathrm{a}$ & $2,91 \mathrm{a}$ & $3,11 \mathrm{a}$ & $4,31 \mathrm{a}$ & $3,82 \mathrm{a}$ \\
$1^{\circ}$ grau completo & $3,36 \mathrm{a}$ & $2,52 \mathrm{a}$ & $3,34 \mathrm{a}$ & $2,99 \mathrm{a}$ & $3,34 \mathrm{a}$ & $4,30 \mathrm{a}$ & $4,07 \mathrm{a}$ \\
$2^{\circ}$ grau completo & $2,91 \mathrm{~b}$ & $2,45 \mathrm{a}$ & $3,06 \mathrm{a}$ & $2,83 \mathrm{a}$ & $3,08 \mathrm{a}$ & $4,20 \mathrm{a}$ & $3,94 \mathrm{a}$ \\
Superior & $2,83 \mathrm{~b}$ & $2,38 \mathrm{a}$ & $3,03 \mathrm{a}$ & $2,71 \mathrm{a}$ & $3,15 \mathrm{a}$ & $4,00 \mathrm{a}$ & $3,71 \mathrm{a}$ \\
Idade & $\mathrm{p}<0,05$ & $\mathrm{p}=0,16$ & $\mathrm{p}=0,24$ & $\mathrm{p}=0,13$ & $\mathrm{p}=0,37$ & $\mathrm{p}<0,05$ & $\mathrm{p}=0,15$ \\
Até 30 & $2,66 \mathrm{c}$ & $2,45 \mathrm{ab}$ & $3,00 \mathrm{a}$ & $2,70 \mathrm{ab}$ & $3,00 \mathrm{ab}$ & $4,00 \mathrm{~b}$ & $3,73 \mathrm{ab}$ \\
Até 40 & $3,12 \mathrm{ab}$ & $2,42 \mathrm{ab}$ & $3,18 \mathrm{a}$ & $2,67 \mathrm{~b}$ & $3,09 \mathrm{ab}$ & $4,06 \mathrm{~b}$ & $3,63 \mathrm{~b}$ \\
Até 50 & $3,21 \mathrm{ab}$ & $2,53 \mathrm{ab}$ & $3,35 \mathrm{a}$ & $2,93 \mathrm{ab}$ & $3,25 \mathrm{ab}$ & $4,21 \mathrm{ab}$ & $4,02 \mathrm{ab}$ \\
Até 60 & $3,48 \mathrm{a}$ & $2,72 \mathrm{a}$ & $3,29 \mathrm{a}$ & $3,09 \mathrm{a}$ & $3,29 \mathrm{a}$ & $4,44 \mathrm{a}$ & $4,05 \mathrm{a}$ \\
$>$ 60 & $2,91 \mathrm{bc}$ & $2,21 \mathrm{~b}$ & $2,96 \mathrm{a}$ & $2,69 \mathrm{ab}$ & $2,94 \mathrm{~b}$ & $4,22 \mathrm{ab}$ & $3,91 \mathrm{ab}$ \\
Estado civil & $\mathrm{p}=0,50$ & $\mathrm{p}=0,43$ & $\mathrm{p}=0,54$ & $\mathrm{p}=0,77$ & $\mathrm{p}=0,44$ & $\mathrm{p}=0,87$ & $\mathrm{p}=0,79$ \\
Solteiro & $2,93 \mathrm{a}$ & $2,45 \mathrm{a}$ & $3,41 \mathrm{a}$ & $2,81 \mathrm{a}$ & $3,21 \mathrm{a}$ & $4,20 \mathrm{a}$ & $3,84 \mathrm{a}$ \\
Casado & $3,05 \mathrm{a}$ & $2,32 \mathrm{a}$ & $3,38 \mathrm{a}$ & $2,76 \mathrm{a}$ & $3,08 \mathrm{a}$ & $4,18 \mathrm{a}$ & $3,80 \mathrm{a}$ \\
Local & $\mathrm{p}<0,05$ & $\mathrm{p}<0,05$ & $\mathrm{p}<0,05$ & $\mathrm{p}=0,07$ & $\mathrm{p}=0,07$ & $\mathrm{p}=0,78$ & $\mathrm{p}<0,05$ \\
Asa Norte (grupo 1) & $2,73 \mathrm{~b}$ & $1,95 \mathrm{c}$ & $3,13 \mathrm{~b}$ & $2,55 \mathrm{~b}$ & $3,15 \mathrm{ab}$ & $4,11 \mathrm{a}$ & $3,41 \mathrm{~b}$ \\
Taguatinga (grupo 2) & $3,52 \mathrm{a}$ & $2,97 \mathrm{a}$ & $3,06 \mathrm{~b}$ & $3,13 \mathrm{a}$ & $3,09 \mathrm{~b}$ & $4,15 \mathrm{a}$ & $4,16 \mathrm{a}$ \\
Riacho Fundo (grupo 3) & $2,89 \mathrm{~b}$ & $2,69 \mathrm{ab}$ & $3,00 \mathrm{~b}$ & $3,00 \mathrm{a}$ & $3,01 \mathrm{~b}$ & $4,21 \mathrm{a}$ & $4,39 \mathrm{a}$ \\
Ceilândia (grupo 4) & $3,54 \mathrm{a}$ & $2,28 \mathrm{bc}$ & $3,92 \mathrm{a}$ & $2,76 \mathrm{ab}$ & $3,52 \mathrm{a}$ & $4,25 \mathrm{a}$ & $3,30 \mathrm{~b}$ \\
Recanto das Emas (grupo 5) & $2,99 \mathrm{~b}$ & $2,66 \mathrm{ab}$ & $2,83 \mathrm{~b}$ & $2,88 \mathrm{ab}$ & $3,03 \mathrm{~b}$ & $4,30 \mathrm{a}$ & $4,18 \mathrm{a}$ \\
\hline
\end{tabular}

Médias seguidas de letras diferentes na coluna diferiram significativamente a $5 \%$ de probabilidade de erro. $\mathrm{UN}=$ uniformidade; $\mathrm{PE}=$ peso; $\mathrm{DI}=$ diâmetro; $\mathrm{FO}=$ formato; $\mathrm{CO}=$ comprimento; $\mathrm{CE}=$ cor externa; $\mathrm{CI}=$ cor interna; Idade $\mathrm{x} F \mathrm{FO}$ : $<<0,05$ 2-4; Idade $\mathrm{x} \mathrm{CO}$ : $\mathrm{p}<0,05$ 4-5; Idade x CI: $\mathrm{p}<0,05$ 2-4; Local x FO: p<0,05 2-3 e 3-4; Local x CO: p<0,05 1-2, 1-4, 1-5 (means followed by different letters in the column differ significantly at $5 \%$ probability. $\mathrm{UN}=$ uniformity; $\mathrm{PE}=$ weight; $\mathrm{DI}=$ diameter; $\mathrm{FO}=$ format; $\mathrm{CO}=$ length; $\mathrm{EC}=$ external color; $\mathrm{CI}=$ internal color; Age x FOR: $p<0.05$ 2-4; Age x CO: $p<0.05$ 4-5; Age x CI: $<<0.05$ 2-4; Local x FO: $p<0.052$ - 3 and 3-4; Local x CO: $p<0.05$ 1-2, 1-4, 1-5).

perceberam melhor a importância do valor nutricional da cenoura. Cabe salientar que ombro verde e uniformidade são mais ressaltados por pessoas com nível de escolaridade de primeiro grau. As pessoas entre 41 a 60 anos são mais criteriosas em relação aos atributos da cenoura do que os jovens e as pessoas acima de 60 anos.

Ao analisar as características sob a ótica da localização dos supermercados, os consumidores do supermercado da Asa Norte (maior poder aquisitivo) dão maior importância ao valor nutricional e os entrevistados do Recanto das Emas (menor poder aquisitivo) preocupam-se mais com preço.

Por fim, a pesquisa sugere que os consumidores estão preocupados com valores nutricionais, textura, cor externa e sabor da cenoura. Já os demais agentes da cadeia atentam mais para as características relacionadas à padronização e à coloração externa. Ombro verde foi valorado porque é considerado dano grave na tabela de classificação da cenoura.

As características valoradas de forma semelhante por todos os agentes da cadeia foram o preço do produto, característica importante e que determina o aumento ou diminuição do consumo; coloração externa e lisura da raiz, que deve ser alaranjada intensa e lisa; caracteres componentes do formato da raiz como formato, diâmetro e comprimento, apresentaram importância mediana. O único carater que recebeu pouca importância para ambos os grupos foi amargor, devido a esta característica não ser comum nas cultivares atuais.

Diferenças de percepção entre os agentes da cadeia do grupo 1 foram pontuadas, destacando a visão dos processadores. Em adição, foram detectados novos olhares quanto a gênero, idade, grau de escolaridade, estado civil e localização do supermercado. Como essa pesquisa analisou o comportamento de um grupo de 66 agentes da cadeia e 500 consumidores do Distrito Federal, a extrapolação necessitará de estudos adicionais para generalizar os resultados. Propõe-se, no futuro, realizar um trabalho com um grupo maior e de outros estados para explorar a diferença de perfil dos agentes da cadeia, incluindo os consumidores. Estes resultados trazem nova luz para o direcionamento de projetos de pesquisa com cenoura, principalmente no que se refere ao melhoramento genético desta hortaliça, que precisa atender às exigências tanto do setor produtivo quanto dos consumidores. 
Tabela 2b. Importância dos atributos da cenoura em raiz segundo o perfil dos agentes da cadeia do grupo 2 (importance of the carrot root attributes according to the profile of the chain agents of the group 2). Brasília, Embrapa Hortaliças, 2009.

\begin{tabular}{|c|c|c|c|c|c|c|c|}
\hline Perfil & $\mathbf{O V}$ & LR & DO & $\mathbf{A M}$ & $\mathbf{C R}$ & VT & PR \\
\hline Sexo & $\mathrm{p}=0,06$ & $\mathrm{p}<0,05$ & $\mathrm{p}=0,31$ & $\mathrm{p}=0,05$ & $\mathrm{p}<0,05$ & $\mathrm{p}=0,11$ & $\mathrm{p}=0,33$ \\
\hline Masculino & $2,13 \mathrm{a}$ & $4,00 \mathrm{a}$ & $3,90 \mathrm{a}$ & $2,38 \mathrm{a}$ & $4,16 b$ & $4,40 \mathrm{a}$ & $3,83 \mathrm{a}$ \\
\hline Feminino & $2,42 \mathrm{a}$ & $3,66 b$ & $4,03 \mathrm{a}$ & $2,69 \mathrm{a}$ & $4,42 \mathrm{a}$ & $4,57 \mathrm{a}$ & $3,97 \mathrm{a}$ \\
\hline Escolaridade & $\mathrm{p}<0,05$ & $\mathrm{p}=0,33$ & $\mathrm{p}=0,12$ & $\mathrm{p}=0,20$ & $\mathrm{p}=0,08$ & $\mathrm{p}<0,05$ & $\mathrm{p}=0,33$ \\
\hline $1^{\circ}$ grau incompleto & 2,39ab & $3,80 \mathrm{a}$ & $4,05 \mathrm{ab}$ & $2,89 \mathrm{a}$ & $4,51 \mathrm{a}$ & $4,38 b$ & $4,07 \mathrm{a}$ \\
\hline $1^{\circ}$ grau completo & $2,63 \mathrm{a}$ & $3,86 \mathrm{a}$ & $4,20 \mathrm{a}$ & $2,52 \mathrm{ab}$ & $4,34 \mathrm{ab}$ & $4,40 \mathrm{~b}$ & $3,88 \mathrm{a}$ \\
\hline $2^{\circ}$ grau completo & $2,28 \mathrm{ab}$ & $3,84 \mathrm{a}$ & $3,97 \mathrm{ab}$ & $2,46 b$ & $4,16 b$ & $4,54 \mathrm{ab}$ & $3,97 \mathrm{a}$ \\
\hline Superior & $1,99 b$ & $3,51 \mathrm{a}$ & $3,76 b$ & $2,48 \mathrm{ab}$ & $4,44 \mathrm{ab}$ & $4,70 \mathrm{a}$ & $3,69 \mathrm{a}$ \\
\hline Idade & $\mathrm{p}<0,05$ & $\mathrm{p}<0,05$ & $\mathrm{p}=0,22$ & $\mathrm{p}=0,13$ & $\mathrm{p}=0,80$ & $\mathrm{p}=0,41$ & $\mathrm{p}=0,78$ \\
\hline Até 30 & $1,99 b$ & $3,6 b c$ & $4,06 \mathrm{ab}$ & $2,17 b$ & $4,19 \mathrm{a}$ & $4,49 a$ & $3,93 \mathrm{a}$ \\
\hline Até 40 & $2,10 \mathrm{~b}$ & $3,39 \mathrm{c}$ & $3,89 b$ & $2,71 \mathrm{a}$ & $4,42 \mathrm{a}$ & $4,57 \mathrm{a}$ & $3,97 \mathrm{a}$ \\
\hline Até 50 & $2,25 b$ & $3,84 \mathrm{ab}$ & $4,19 \mathrm{a}$ & $2,68 \mathrm{a}$ & $4,37 \mathrm{a}$ & $4,63 \mathrm{a}$ & $3,76 a$ \\
\hline Até 60 & $2,79 \mathrm{a}$ & $4,02 \mathrm{a}$ & $3,82 b$ & $2,66 \mathrm{a}$ & $4,34 \mathrm{a}$ & $4,40 \mathrm{a}$ & $4,00 \mathrm{a}$ \\
\hline$>60$ & $2,28 b$ & $3,91 \mathrm{ab}$ & $3,97 \mathrm{ab}$ & $2,60 \mathrm{ab}$ & $4,30 \mathrm{a}$ & $4,46 a$ & $3,99 \mathrm{a}$ \\
\hline Estado civil & $\mathrm{p}=0,75$ & $\mathrm{p}=0,10$ & $\mathrm{p}=0,07$ & $\mathrm{p}=0,20$ & - & $\mathrm{p}<0,05$ & $\mathrm{p}=0,47$ \\
\hline Solteiro & $2,28 \mathrm{a}$ & $3,77 \mathrm{a}$ & $4,06 \mathrm{a}$ & $2,47 a$ & - & $4,68 \mathrm{a}$ & $4,11 \mathrm{a}$ \\
\hline Casado & $2,22 \mathrm{a}$ & $3,46 \mathrm{a}$ & $3,76 \mathrm{a}$ & $2,72 \mathrm{a}$ & - & $4,42 b$ & $3,99 \mathrm{a}$ \\
\hline Local & $\mathrm{p}<0,05$ & $\mathrm{p}<0,05$ & $\mathrm{p}<0,05$ & $\mathrm{p}<0,05$ & $\mathrm{p}<0,05$ & $\mathrm{p}<0,05$ & $\mathrm{p}<0,05$ \\
\hline Asa Norte (grupo 1) & $2,22 b$ & $3,30 \mathrm{c}$ & $3,51 \mathrm{~b}$ & $3,03 b$ & $4,37 \mathrm{a}$ & $4,74 a$ & $3,49 \mathrm{c}$ \\
\hline Taguatinga (grupo 2) & $2,54 \mathrm{ab}$ & $4,31 \mathrm{a}$ & $4,41 \mathrm{a}$ & $2,12 \mathrm{c}$ & $4,38 \mathrm{a}$ & $4,66 \mathrm{ab}$ & $3,44 \mathrm{c}$ \\
\hline Riacho Fundo (grupo 3) & $2,17 b$ & $3,83 b$ & $4,21 \mathrm{a}$ & $2,05 \mathrm{c}$ & $4,07 \mathrm{a}$ & $4,54 \mathrm{ab}$ & $4,26 \mathrm{ab}$ \\
\hline Ceilândia (grupo 4) & $2,93 \mathrm{a}$ & $3,15 \mathrm{c}$ & $3,53 b$ & $3,97 \mathrm{a}$ & $4,38 \mathrm{a}$ & $4,09 \mathrm{c}$ & $3,99 b$ \\
\hline Recanto das Emas (grupo 5) & $1,76 \mathrm{c}$ & $4,26 \mathrm{a}$ & $4,24 \mathrm{a}$ & $1,74 \mathrm{c}$ & $4,39 a$ & $4,47 b c$ & $4,42 \mathrm{a}$ \\
\hline
\end{tabular}

Médias seguidas de letras diferentes na coluna diferiram significativamente a $5 \%$ de probabilidade de erro. $\mathrm{OV}=$ ombro verde; $\mathrm{LR}=$ lisura na raiz; $\mathrm{DO}=$ doçura; $\mathrm{AM}=$ amargor; $\mathrm{CR}=$ crocância; $\mathrm{VT}=$ vitamina; $\mathrm{PR}=$ preço; Escolaridade $\mathrm{x} \mathrm{DO}$ : $\mathrm{p}<0,05$ 2-4; Escolaridade $\mathrm{x} A M$ : $\mathrm{p}<0,05$ 1-3; Escolaridade x CR: p<0,05 1-3; Idade x DO: $<<0,05$ 3-4; Idade x AM: p<0,05 1-2, 1-3, 1-4 (means followed by different letters in the column differ significantly at $5 \%$ probability. $\mathrm{OV}=$ green shoulder; $\mathrm{LR}=$ root smoothness; $\mathrm{DO}=$ sweetness; $\mathrm{AM}=$ bitterness; $\mathrm{CR}=$ crispness; $\mathrm{VT}=$ vitamin; $\mathrm{PR}=$ price; Scholar $\mathrm{x}$ DO: $\mathrm{p}<0.05$ 2-4; Scholar $\mathrm{x}$ AM: $<<0.05$ 1-3; Scholar $\mathrm{x}$ CR: $\mathrm{p}<0.05$ 1-3; Age x DO: $\mathrm{p}<0.05$ 3-4; Age $\mathrm{x}$ AM: $\mathrm{p}<0.05$ 1-2, 1-3, 1-4). ${ }^{1}$ Não foi possível fazer o contraste de crocância com estado civil, porque foram menos que cinco estratificações

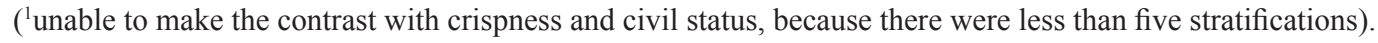

\section{AGRADECIMENTOS}

A todos os agentes da cadeia entrevistados e ao Dr. Nozomu Makishima pela orientação e auxilio nas entrevistas ocorridas no estado de São Paulo.

\section{REFERÊNCIAS}

BLACKWELL RD; MINIARD JF; ENGEL. 2005. Comportamento do Consumidor. Rio de Janeiro: Cengage Learning, 606p.

BRENNAN J; BYERLEE D. 1991. The rate of crop varietal replacement on farms: measures and empirical results for wheat. Plant Var Seeds 4: 99-106.

CICCANTELLI S; MAGIDSON J. 1993. From experience: Consumer idealized design-involving consumers in the product development process. Journal of Product Innovation Management 10: 341-347.
CLARK K; WHEELWRIGHT SC. 1993. Managing New Product and Process Development: Test and Cases. New York: Fee Press, 896p.

COOPER RG. 1999. From Experience: The invisible success factors in produt innovation. Journal of Product Innovation Management 16: 115-133.

CODEPLAN. 1997. Pesquisa de Informações Sócio-Econômicas das Famílias do Distrito Federal. Brasília: PISEF/DF, 120p.

KADER AA. 2002. Postharvest Technology of Horticultural Crops. California: Ed. University of California: Division of Agriculture and Natural Resources Publication, 535p.

KOSINA P; REYNOLDS M; DIXON J; JOSHI A. 2007. Stakeholder perception of wheat production constraints, capacity building needs, and research partnerships in developing countries. Euphytica 157: 475-483.

MATSUURA FCAU; COSTA JIP; FOLEGATTI MIS. 2004. Marketing de banana: preferências do consumidor quanto aos atributos de qualidade dos frutos. Revista Brasileira de Fruticultura 26: 48-52.

MATTAR FN. 1997. Pesquisa de marketing: metodologia, planejamento. 4. ed. São Paulo: Atlas. 336p.

SOUZA RAM. 2005. Mudanças no consumo e na distribuição de alimentos - $O$ caso da distribuição de hortaliças de folhas na cidade de São Paulo. Campinas: UNICAMP-IE. 150p. (Tese mestrado).

STOKES ME; DAVIS CS; KOCH GG. 2000. Categorical Data Analysis Using SAS System. Second Edition. Cary, NC: SAS Institute Inc, $623 p$.

VILELA NJ; BORGES IO. 2008. Retrospectiva e situação atual da cenoura no Brasil. Brasília: Embrapa Hortaliças, 9p. (Circular Técnica, 59).

VILELA NJ; HENZ GP. 2000. Situação atual da participação das hortaliças no agronegócio brasileiro e perspectivas futuras. Cadernos de Ciências e Tecnologia 17: 71-89. 